\title{
A neurologically plausible implementation of statistical inference applied to random dot motion James Martens* ${ }^{* 1}$ and Chris Eliasmith ${ }^{1,2}$
}

\author{
Address: ${ }^{1}$ Cheriton School of Computer Science University of Waterloo, Waterloo, Canada and ${ }^{2}$ Department of Philosophy, Department of \\ Systems Design Engineering, University of Waterloo, Waterloo, Canada \\ Email: James Martens* - james.martens@gmail.com \\ * Corresponding author
}

from Sixteenth Annual Computational Neuroscience Meeting: CNS*2007

Toronto, Canada. 7-12 July 2007

Published: 6 July 2007

BMC Neuroscience 2007, 8(Suppl 2):P207 doi: I0.I | 86/ | 47|-2202-8-S2-P207

(C) 2007 Martens and Eliasmith; licensee BioMed Central Ltd.

We propose a model of basic motion perception consisting of a hierarchical non-linear state space model (NSSM) developed within a variational Bayesian (VB) framework. Each level of the hierarchy is a 'cause' that generates a prior distribution on the level below via a generative function.

The temporal dynamics and generative functions between layers of the hierarchy are implemented as neural networks with non-linear activation functions. Optimization of model parameters and causes proceed concurrently as a combination of fixed-point rules and gradient decent schemes. To make the optimization problem tractable, the standard mean-field and Laplace approximations are employed. The precise factoring used in the mean-field approximation is designed to meet a balance between tractability, neurological plausibility and modeling power. In this approach inference and learning proceed concurrently, in an online and unsupervised fashion.

Other work has produced similar implementations of NSSMs that have been successful in predicting lowdimensional temporal signals, but with highly restrictive assumptions on the form of the posterior distributions and with learning done in batches instead of online [1].

Competing work has relaxed many of these implementation assumptions and achieved prediction of high dimensional input but at the cost of discarding VB techniques in favor of inefficient discrete state-space methods [2]. More- over, such techniques have been demonstrated only with pre-learned weights and simplistic statistical models.

Our model is primarily tested on realistic high-dimensional input generated by randomly moving dots over a detection grid. The results from a spiking neuron implementation of the model based on the Neural Engineering Framework (NEF) are compared directly to single cell recordings in random dot motion perception and decision-making tasks.

\section{References}

I. Valpola $H$, Karhunen J: An unsupervised ensemble learning method for nonlinear dynamic state-space models. Neural Computation 2002, I 4( I I):2647-2692.

2. Rao R: Hierarchical Bayesian Inference in Networks of Spiking Neurons. NIPS 2005, I7:. 